\title{
Anstabil artrozlu dizde kombine tedavi: proksimal tibial osteotomi ve ön çapraz bağ rekonstrüksiyonu
}

\section{Combined treatment in the knee with unstable arthrosis: proximal tibial osteotomy and anterior cruciate ligament reconstruction}

\author{
Nurzat Elmalı, Vahdet Uçan, Muzaffer Ağır
}

Bezmialem Vakıf Üniversitesi Tıp Fakültesi Ortopedi ve Travmatoloji Anabilim Dalı, İstanbul

Ön çapraz bağ (ÖÇB) yetersizliğine bağlı kronik anterior laksite tibiofemoral osteoartrit (OA) ve sıklıkla varus dizilim bozukluğu ile birliktedir. İzole ÖÇB rekonstrüksiyonu bu durumlarda anterior diz instabilitesini iyileştirir ancak OA'nın ilerlemesini önlemez. Proksimal tibial osteotomi (PTO) genç-orta yaşlı hastalarda varus dizilim bozukluğu ile birlikte olan medial kompartman osteoartritinin tedavisinde kabul edilen bir yöntemdir ve anstabil artritli dizde de diz stabilitesini yeniden sağlamada önemli bir rol oynar. Varus OA’lı kronik ÖÇB yetersizliği, hastanın yaşı, aktivite düzeyi ve instabilite semptomlarının olup olmayışına göre PTO ile tek başına veya ÖÇB rekonstrüksiyonu ile kombine tedavi edilir. PTO, anstabil artritik dizlerde varus dizilimini düzeltir, medial kompartmanı yükten kurtarır ve ligament rekonstrüksiyonunun sonuçlarını iyileştirir.

Anahtar sözcükler: diz; ligament; instabilite; varus; artroz; osteotomi
Chronic anterior laxity due to anterior cruciate ligament ( $A C L)$ deficiency is associated with the development of tibiofemoral osteoarthritis (OA) and frequently varus malalignment. Isolated $A C L$ reconstruction (ACLR) can restore anterior knee stability in such cases, but does not mitigate progression of OA. Proximal tibial osteotomy (PTO) has long been an accepted treatment for medial compartment $\mathrm{OA}$ with varus malalignment in young and middle age patients and also plays a large role in knee restoring knee stability in the setting of unstable arthritic knee. Chronic ACL injury with varus osteoarthritis should be treated with $\mathrm{HTO}$ alone or $\mathrm{HTO}$ combined with $\mathrm{ACL}$ reconstruction, depending on the patient's age, activity level and the reported instability. Proximal tibial osteotomy corrects the varus alignment and offloads the medial compartment and improves the result of ligament reconstruction.

Key words: knee; ligament; instability; varus; arthrosis; osteotomy enç sporcularda ön çapraz bağ (ÖÇB) yetersizliği iyi tolere edilemez ve kronik instabiliteye bağlı gelişen kıkırdak ve menisküs yaralanmaları posttravmatik osteoartrite ve varus dizilim bozukluğuna neden olur. ${ }^{[1,2]}$

Hastaların genç yaşta olması ve spora devam etmedeki yüksek beklentileri nedeniyle tedavileri güçtür. $\mathrm{Bu}$ özel grup hastaları etkili tedavi etmek için bağ yetersizliği olan dizde artrozun nasıl geliştiğini ve ameliyata karar verirken değerlendirilmesi gereken parametreleri iyi belirlemek gerekir.

ÖÇB kopuk dizlerde tibianın aşıı öne translasyonu sonucu olarak eklem kıkırdağına, menisküslerin özellikle posterior kısmına ve ligamentlere gelen yüklenmelerin artmasına bağlı fleksiyonda femoral kayma ve yuvarlanma hareketinin fizyolojik oranları bozulur. ${ }^{[3]}$ ÖÇB kopmasında akut menisküs yırtığı \%52 sıklıkta görülürken kronik vakalarda \%83'e çıkar. ${ }^{[4]}$ Gecikmiş ÖÇB rekonstrüksiyonu yapılan hastaların yarıdan fazlasına menisektomi yapıldığı bildirilmiş̧tir. ${ }^{[5]}$ Menisektomi erken başlangıçı ı medial osteoartrit için kesin risk faktörüdür. ${ }^{[3]}$

Diz instabilitesinin tedavisinde başarıyı etkileyen önemli faktörlerden biri dizilimin dikkate alınmasıdır. Varus dizilimi yapısal (constitutional) olabilir veya sekonder olarak medial osteokondral lezyonun genişlemesi ile, menisküs yırtığı veya menisektomi sonrası oluşabilir. ÖÇB, diz ekleminin lateral kapsülo-ligamentöz yapılarından sonra gelen ikincil varus sınırlayıcısı olduğu için ÖÇB kopuk dizlerde de varus deformitesi oluşabilmektedir. ${ }^{[6]}$

- İletişim adresi: Prof. Dr. Nurzat Elmalı, Bezmialem Vakıf Üniversitesi Hastanesi Ortopedi ve Travmatoloji Kliniği, Adnan Menderes Bulvarı, Vatan Cad. 34093 Fatih, İstanbul Tel: 0532 - 4359129 e-posta: nelmali@hotmail.com

- Geliștarihi: 18 Subat $2020 \quad$ Kabul tarihi: 4 Mart 2020 
Tablo 1. Primer varus, Double (ikili) varus, Triple (üçlü) varus

Primer Varus (yapısal=constitutional varus)

Double (ikili) varus

Triple (üçlü) varus
Fizyolojik tibiofemoral varus dizilim, ÖÇB yetersizliği ve medial tibiofemoral kompartmanda daralma (medial menisküs ve medial tibiofemoral eklemde kıkırdak kaybı).

Lateral ligamentöz yapılarda gevşemeye bağlı lateral tibiofemoral kompartmanda açılma ile birlikte varus dizilim (lateral condylar lift-off). "Varus thrust" yürüyüş, yürümenin yük verme (stance) fazında belirginleşen dinamik varus.

Zorlamalı grafilerde medial eklem aralığında daralma, lateral eklem mesafesinde 5 mm'den fazla açılma görülür.

Kronik gerim etkisinde tüm posterolateral ligament kompleksinin ciddi etkilenmesi ile tibiofemoral varus dizilim, dizde artmış dış rotasyon, anormal varus rekurvatum pozisyonu ve lateral tibiofemoral kompartmanda $12 \mathrm{~mm}$ 'den fazla ayrılma
ÖÇB yetersizliği ile birlikte primer veya sekonder varus dizilim bozukluğunun olması adduktör momentin artışı ve nöromuskuler kontrolün kaybı nedeniyle hem varusu hem de medial kompartmandaki yüklenmeyi daha da artırır ve varus itme yürüyüşü (double varus=ikili varus=varus thrust) oluşur. Kronik instabilite sonucu medial kıkırdak hasarının artması ve varusun ilerlemesi ile lateral eklem aralığında açılma, posterolateral yapılarda uzama ve gevşeme olur. Klinik olarak ekstansiyonda varus rekurvatum pozisyonu ile birlikte ciddi posterolateral yetersizlik gelişir (triple varus=üçlü varus) (Tablo 1). ${ }^{[7-9]}$

Sonuç olarak dizde kronik instabilite, önceden var olan (yapısal) veya sonradan gelişen varus dizilim bozukluğu ile birlikte olduğunda medial unikompartmantal osteoartrite neden olur. ${ }^{[10]}$ Kronik ÖÇB yetersizliği ve medial kompartman osteoartriti olan hastalar ya tek başına proksimal tibial osteotomi ile veya kombine eş zamanlı veya aşamalı proksimal tibial osteotomi (PTO) ve ÖÇB rekonstrüksiyonu (ÖÇBR) ile tedavi edilir. ÖÇBR ile birlikte PTO yapılmasının uzun dönem izlemde iyi-mükemmel sonuçlarla artritin ilerlemesini yavaşlattığı bildirilmiştir. ${ }^{[11-17]}$ ÖÇBR anterior tibial translasyonu kontrol ederek diz biyomekaniklerini iyileştirirken PTO medial kompartmanı yüklenmesiz bırakarak osteoartritin ilerlemesini geciktirebilir. Genel olarak eş zamanlı kombine cerrahi, teknik güçlüklerine rağmen total cerrahi süresini fazla artırmayan kısa süreli işlem olması ve hastaların daha erken iyileşmesine imkân vermesi bakımından tercih edilir. İki aşamalı cerrahi planlandığında önce osteotomi yapılır. İnstabilite devam ederse 6-12 ay sonra ÖÇBR yapılır.

\section{Ameliyat Öncesi Değerlendirme}

Semptomlar genellikle ÖÇB yetersizliğine bağlı instabilite, medial kompartman artrozundan orijin alan ağrı, varus ve hiperekstansiyon yürüyüşü ile ilişkilidir.

ÖÇB yetersizliği ve medial kompartman artritinin birlikte olduğu hastalarda esas şikâyetin ayırt edilmesi önemlidir. Hasta yakınmaları ağırlıklı olarak instabiliteden mi yoksa dejeneratif artritten mi kaynaklanmaktadır? ÖÇB yetersizliğinin doğal seyri sırasında ağrı ve instabilite farklı yolla meydana gelir. ÖÇB kopmasından sonra instabilite en önemli parametredir. Ağrı başlangıçta önemli değildir ancak zamanla dejeneratif süreç ilerledikçe ve artritik değişiklikler meydana geldikçe dizdeki instabilite azalır ve ağrı ön plana geçer (Şekil 1).

Muayene, alt ekstremite diziliminin, yürüyüşün, eklem hareket açıklığının ve stabilitenin değerlendirilmesini içermelidir. İnstabilite, artroz veya kombine instabilite ve artrozu olan hastalarda alt ekstremitenin hem koronal (varus/valgus) hem de sagittal (tibial slop) plan dizilimi; otururken, ayakta ve yürürken olmak üzere dikkatlice değerlendirilmelidir. Öne çekmece, Lachman ve pivot şift testleri ile anterior instabilite degerlendirilir. Ciddi varus dizde lateral ve posterolateral yapılarda yetmezlik olabilir. Varus stres testi, eksternal rotasyon rekurvatum testi, Dial testi ile postero-lateral köşe yetmezliği değerlendirilir.

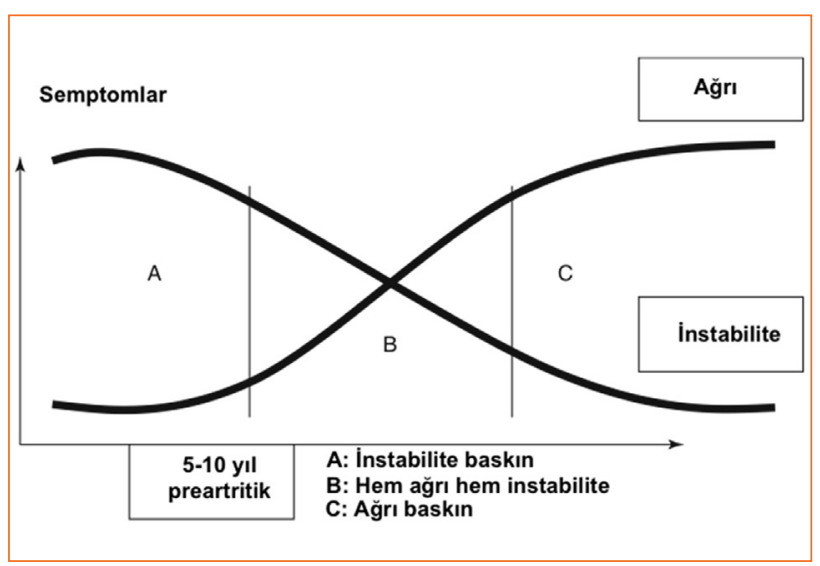

Şekil 1. İnstabilite ve ağrı arasında zamana bağlı ilişki. 
Radyolojik değerlendirmede, ön-arka ve yan grafiler, $45^{\circ}$ fleksiyonda yük vererek ön arka grafi (Rosenberg grafisi), varus ve valgus zorlamalı grafi, ayakta uzun bacak grafisi (ortoröntgenogram) ve manyetik rezonans görüntüleme (MRG) ile alt ekstremite dizilimi, posterior tibial slop, patellar yükseklik, kıkırdak, bağ ve menisküslerin durumu değerlendirilir.

Cerrahi yönteme karar verirken esas olarak hastanın yaşı, aktivite derecesi, primer semptomları ve varus deformitesi dikkate alınır. ÖÇB yetersizliğine bağlı instabilite ile birlikte primer (yapısal=constitutional) varus deformitesinin olması durumunda dizilimin düzeltilmesi gerekmez. Bu kişilerde varus dizilim bozukluğunun düzeltilmesinin ÖÇB cerrahisinin sonuçlarını etkilemediği gösterilmiştir. ${ }^{[18]}$ Orta yaşlı veya daha az aktif hasta yürüme, merdiven çıkma veya namaz kılma gibi günlük aktiviteleri sırasında mekanik aşırı yüklenme ile ağrıdan şikâyetçi ise artroz ön plandadır. Zaman zaman güvensizlik şikâyeti olabilir. Bu hastalarda tek başına osteotomi yeterli olacaktır. Osteotomi sırasında posterior tibial eğimi (slop) azaltma bu hastalar için yeterli stabilite sağlayabilir. ${ }^{[19]}$ Ancak kronik ÖÇB yetersizliği ve varusu olan yüksek beklentili genç hastada günlük veya spor aktiviteleri sırasında güvensizlik oluyorsa instabilite ön plandadır. Ağrı ve hafif-orta derecede radyolojik osteoartrit bulguları olabilir. Bu hastalarda kombine yüksek tibial osteotomi ve ÖÇB rekonstrüksiyonu hem instabilite hem de medial eklem ağrısını gidermek için uygun olacaktır. İnstabilite şikâyetleri olan orta yaşlı aktif hastalarda da osteotomiye ÖÇBR eklenmesine eğilim artmaktadır. ${ }^{[12,13]}$

Yürüme sırasında varus/valgus veya rekurvatumun değerlendirilmesi önemlidir. Yürürken ortaya çıkan varus itme (ikilivarus)'nin olması veya hiperekstansiyon ile birlikte varus itme (üçlü varus)'nin olması durumunda ÖÇBR'ye ilaveten varusun düzeltilmesi başarılı sonuç verecektir. ${ }^{[8,9]}$ Eşlik eden lateral veya posterolateral köşe yaralanması (PLK)'nı belirlemek için klinik ve radyolojik muayenenin dikkatli yapılması önemlidir. Varus zorlamalı grafide lateral kompartmanda $12 \mathrm{~mm}$ 'den daha fazla bir açılma varsa, bağ rekonstrüksiyonu gerektiren posterolateral yetmezlik düşünülmelidir. ${ }^{[18]} \mathrm{Bu}$ hastalarda artroskopi sırasında lateral kompartman açılması tekrar değerlendirilmelidir. Üçlü varusu olan dizlerde tek başına ÖÇB rekonstrüksiyonu yapılırsa grefte aşırı yük binmesinden dolayı greftte kopma veya gevşeme riski yüksektir. Bu durumda PTO ile eş zamanlı ÖÇBR ve PLK rekonstrüksiyonu yapılabilir. ${ }^{[18-23]}$ Üçlü varusu olan dizlerde iki aşamalı cerrahi de yapılabilir. Bu durumda ilk seansta PTO yapılır. Hastalar 6-8 ay sonra yeniden değerlendirilir. Eğer hâlâ instabilite devam ediyorsa ikinci aşamada implant çıkartılırken ÖÇBR ve PLKR birlikte uygulanır. ${ }^{[22,23]}$

Son zamanlarda ÖÇB yetersizliği olan dizlerde sagittal plan deformitesine dikkat çekilmektedir. İnstabil dizde posterior tibial slop (PTS)'un değerinin yüksek olmasının femur kondillerine göre tibial platoda anterior ve superior translasyona neden olarak anterior instabiliteyi artırdığı ve rekonstrüksiyon yapılan dizlerde greftin gevşemesine ve yetersizliğine neden olabildiği bildirilmiştir. ${ }^{[24-26]}$ ÖÇB yetersizliği olan ve PTS $12^{\circ}$ 'den büyük olan hastalarda revizyon ÖÇBR ile birlikte posterior tibial slopu azaltmak için anterior kapalı kama osteotomisi önerilmektedir. Özellikle birkaç kez başarısız ÖÇB rekonstrüksiyonu yapılan ve yetersizlik için teknik hata bulgusu olmayan vakalar için dikkate alınmalıdır (Şekil 2). ${ }^{[26]}$

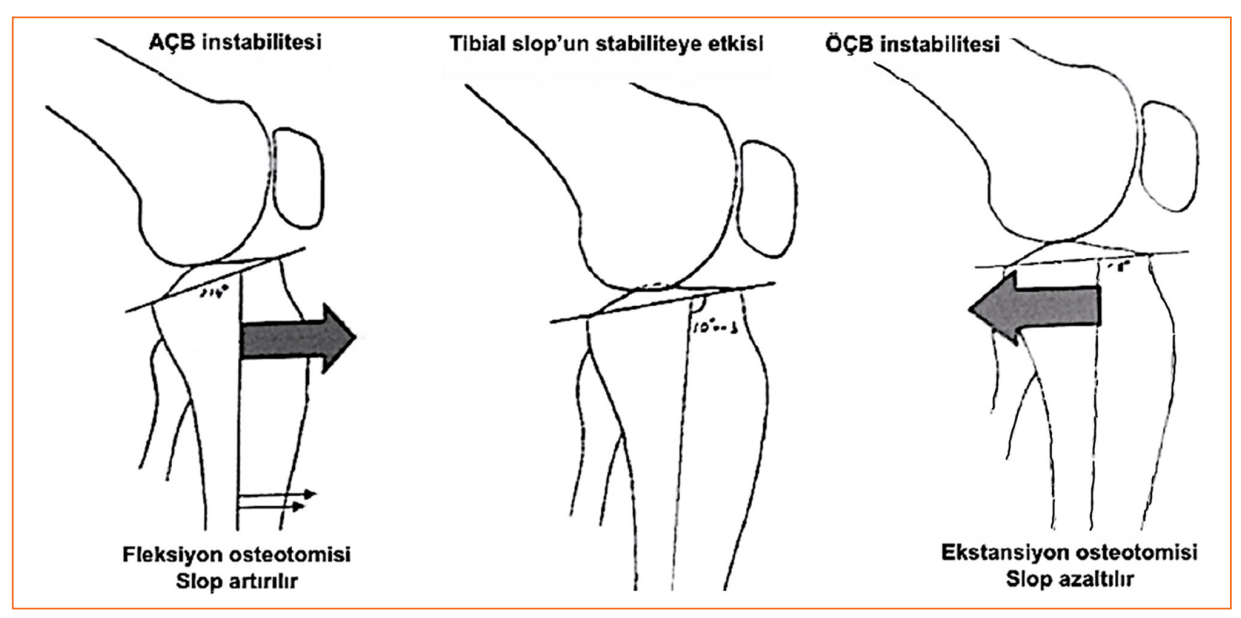

Şekil 2. Tibial eğim (slop) ile tibial translasyon arasındaki ilişki. 
Ameliyat öncesi planlamada ortoröntgenogramlar önemlidir. Düzeltmenin amacı alttaki patolojiye göre farklı olabilir. ÖÇB yetersizliği ile birlikte varus dizilim bozukluğu ve varus itme yürüyüşü (varus thrust) olan hastada nötral bacak dizilimini sağlama amaçlanır. Yüksek aktivite düzeyine yeniden dönmek isteyen aktif hastalarda aşırı düzeltilmiş bacak dizilimi, spora yeniden dönmelerini olumsuz etkileyebilir. Varus dizilim bozukluğu ve medial unikompartmantal osteoartriti olan hastada ise tibial platonun \%62'sinden geçecek şekilde valgusa düzeltme amaçlanır. Radyolojik olarak ortoröntgenogramda femur başı merkezini ayak bileği merkezine birleştiren bir hat (Mickuliz hattı) ile femur başı merkezinden tibia platosunun mediolateral planda \%62'sinden geçen (Fujisawa noktası) bir hat çizilir. $\mathrm{Bu}$ nokta lateral tibial eminensiyaya hafif lateraldir. Proksimalde osteotominin lateral korteksine uzanan menteşe noktası belirlenir. Her iki hat distal ayak bileği seviyesinden proksimale menteşe noktasına uzatılır. Oluşan açı düzeltme miktarını gösterir (Şekil 3).. ${ }^{[20]}$

İnstabil dizde tek başına PTO veya kombine cerrahi endikasyonları tabloda gösterilmiştir (Tablo 2). Bizim de tercih ettiğimiz yöntem olan tek aşamalı cerrahinin komplikasyonları yüksektir ve teknik olarak güçtür. Ancak teknik özelliklere dikkat edildiğinde oldukça başarılıdır.

\section{Eşzamanlı medial açık kama osteotomisi ve ÖÇB rekonstrüksiyonu cerrahi teknik}

1. Hasta pozisyonu ve insizyon: Hasta supin pozisyonda ve normal masada hazırlanır. Uyluğa yüksek seviyede turnike ve lateral post yerleştirilir. Masanın ayağı $120^{\circ}$ diz fleksiyonuna izin verecek şekilde ayarlanır. Grefti hasarlamaktan kaçınmak için önce PTO yapılır. Tibial tüberkülün medial sınırı ve tibianın posteromedial sınırı arasında orta hatta medial eklem hattının $1 \mathrm{~cm}$ aşağısından başlayarak 7-8 cm longitudinal insizyon yapılır.

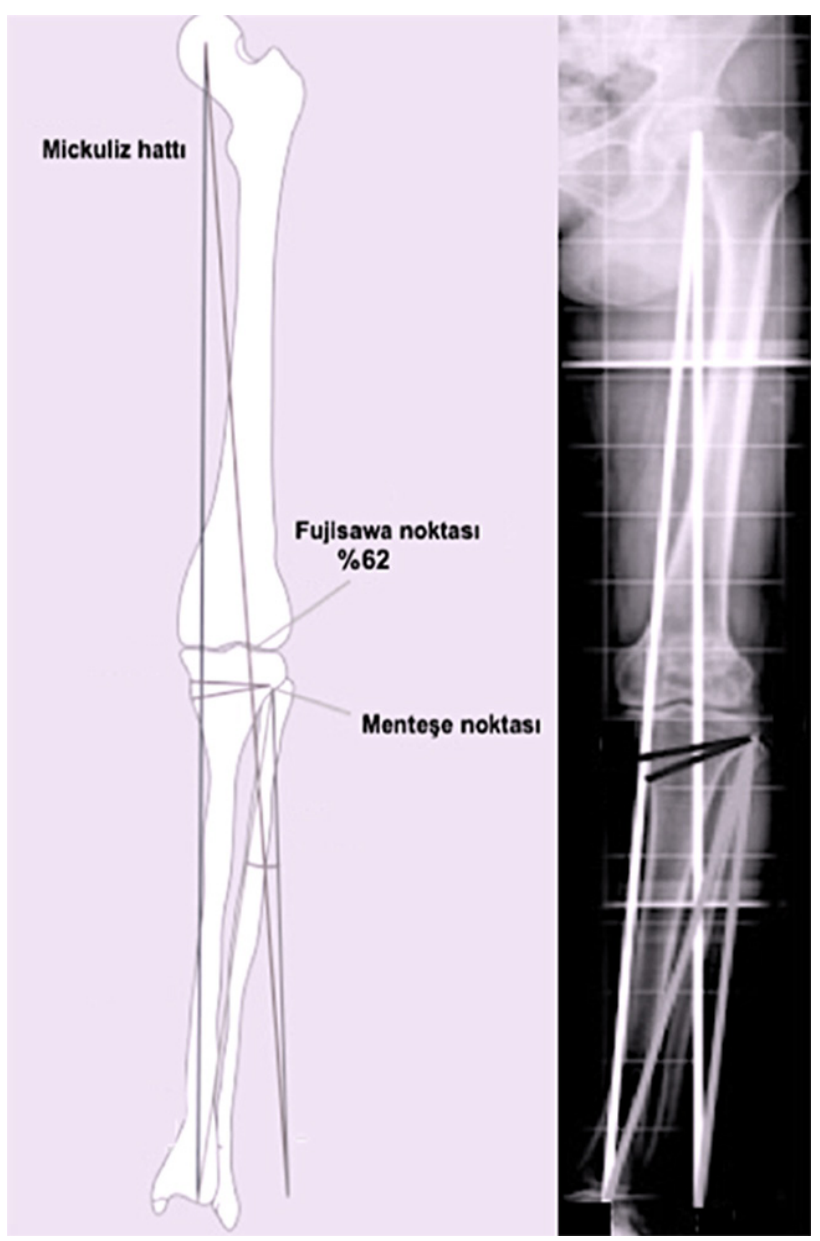

Şekil 3. Düzeltme açısının hesaplanması.

2. Hamstring tendon greftinin alınması: $\mathrm{Bu}$ aşama diz fleksiyonda iken yapılır. Daha önce hamstringler alındıysa allogreft tendon kullanımı tercih edilir.

Tablo 2. Kombine PTO ve ÖÇBR veya revizyonu endikasyonları

\footnotetext{
ÖÇB yetersizliği + varus + medial kompartman osteoartriti (Ahlback Evre 1-3)

Yetersiz ÖÇBR + varus + medial kompartman osteoartriti (Ahlback Evre 1-3)

ÖÇB yetersizliği +ikili veya üçlü varus

ÖÇB yetersizliği + varus + kıkırdak veya menisküs yaralanması (kıkırdak onarımı veya menisküs transplantı gerektiren)

Artmış tibial slop nedeniyle ÖÇBR yetersizliği

İzole PTO (rezidüel instabilite devam ederse gecikmiş ÖÇBR) endikasyonları

Kronik ÖÇB yetersizliği + ikili veya üçlü varus

Kronik ÖÇB yetersizliği + varus + medial kompartman osteoartriti

Kronik ÖÇB yetersizliği + ikili veya üçlü varus + medial kompartman osteoartriti
} 
3. Medial açık kama osteotomisi: Diz $45-60^{\circ}$ fleksiyonda olacak şekilde, bacağın altına gerekirse ayrı bir el masası yerleştirilerek osteotomi yapılır. Önce hamstring greftinin alındığı yerde medial kollateral ligaman (MKL)'ın distal yüzeyel lifleri Cobb elevatörü ile subperiosteal kaldırılır. Bu gevşetme medial kompartmanda aşıı yüklenmenin ortaya çıkmasını önler. Posterior nörovaskuler yapıları korumak için MKL'ye derin künt Hohmann retraktörü yerleştirilir. Kılavuz tellerini göndermeden önce osteotomi hattının açılması sırasında ekleme uzanan kırık oluşmasını önlemek için proksimal tibiada eklem hattının $1 \mathrm{~cm}$ aşağısından iki adet Kirschner $(K)$ teli ekleme paralel gönderilir. Osteotomiye kılavuz olması için eklem hattının $4-5 \mathrm{~cm}$ distalinden skopi kontrolünde medialden laterale fibula başına doğru K teli gönderilir. Bu K teli, ÖÇB tibial tünelinin osteotomi hattının proksimalinde kalması için orijinal osteotomi hattına göre biraz daha distal ve oblik gönderilir. Illk $K$ teline paralel iki adet $K$ teli daha gönderilir. Osteotomi bu $\mathrm{K}$ tellerinin hemen altından yapılır. Medial distalden lateral proksimale fibula başına doğru skopi kontrolü altında lateral korteksi koruyarak ince motorlu testere ile oblik osteotomi yapılır. Lateral korteksin $1 \mathrm{~cm}$ 'si içinde kalınır. Biplanar (iki planlı) osteotomide anterior kesi, esas osteotomiye yaklaşık $110^{\circ}$ açıda ve 1,5 $\mathrm{cm}$ genişliğinde, tüberkül fragmanı kalacak şekilde yapılır. Sagittal planda osteotomi tibial slopa paralel olmalıdır ( $10^{\circ}$ ön-arka eğim ile). Proksimal (Yüksek) tibial osteotomi ile koronal planda varus dizilimi düzeltilirken ÖÇB veya arka çapraz bağ (AÇB) yetersizliği olan dizlerde osteotomi sırasında PTS ayarlanabilir. Açık kama osteotomisi yapılacaksa slopu artırmamak için osteotominin anterior açıklığı posterior açıklığının yarısı kadar olmalıdır. Anterior açıklıktaki her $1 \mathrm{~mm}$ artış PTS'de $2^{\circ}$ artışa neden olur. ${ }^{[15]}$ ilk osteotomun altından osteotomi hattına iki veya üç osteotom daha ilerletilir. Ardından distraktörle hesaplanan düzeltme miktarı sağlanıncaya kadar osteotomi hattı açılır.

4. Osteotominin tamamlanmasından sonra rijit internal fiksatör plak ile osteosentez: (örn., Tomofix; Clinical House, Bochum, Germany). Kombine girişimlerde PTO'nun tespiti için kilitli vidaların yerleştirilmesine izin veren plaklar tercih edilmelidir. Plak mümkün olduğunca posterior olarak yerleştirilerek, tibial tüneli drilleme için anteromedial tibiada daha fazla yer bırakılır. Plağın en proksimal deliklerinin tibial tünel ile çakışmamasına dikkat edilir. Proksimal anteriordaki ilk vida dışındaki tüm vidalar yerleştirilir. Çoğu zaman proksimal anteriordaki vida deliği tünel ile çakışır. Grefti geçirmeden önce açılan tibial tünel içerisinden artroskop ile bakılarak eğer vida tünelin içerisinden geçiyorsa boş bırakılır. Plağın uygun yerleşimi ile diğer vidaların tibial tünel ile çakışması beklenmez. Osteotomi boşluğuna kemik greft konulabilir. Osteosentezin tamamlanmasından sonra hamstringlerle ÖÇB rekonstrüksiyonuna başlanır.

5. Artroskopi ve femoral tünelin hazırlanması: Standart anteromedial ve anterolateral portaller ile artroskopi yapılır. Femoral tünelin anteromedial açılması tibial tünel için daha fazla yer bırakır. Eşlik eden menisküs ve kıkırdak yaralanmaları tedavi edilir. Bizim tercih ettiğimiz yöntem içten dışa (inside-out) transportal femoral tünel drillemedir. Femoral tünelin açılması osteotomiye başlamadan önce de yapılabilir.

6. Tibial tünelin hazırlanması: Tespitin kolaylığı ve daha güvenli olması bakımından tibial tünelin distal çıkışının proksimal fragmanda ve plağın önünde olmasına dikkat edilir. Metal kemik tünel genişletici tibial tünele yerleştirilir ve yerinde bırakılır. İmplantın medial proksimal vidası tibial tünel içerisinden geçebilir. Gerekirse vida çıkarılır ve dril deliklerini etkilemeyen daha kısa vida kullanılabilir.

7. Greftin uygun gerginlikte tünellerden geçirilmesi ve tespiti: Greftin femura tespitinde genellikle ekstrakortikal suspensiyon uygulanır (Endobutton; Smith \& Nephew). Tibial tünel içerisinde biointerferens vida ve tibial tünele distal staple ile greft tespit edilir.

8. Rehabilitasyon süreci: Rehabilitasyon sürecini eklem içi patolojiler ve osteotominin stabilitesi belirler. Açı stabil plak superior stabilite sağladığından tercih edilir. Ek patoloji yoksa cerrahiyi takiben parmak ucu yüklenmeye izin verilir. Dört ila altı haftada tam yüklenmeye izin verilir. ROM (Range of Motion) artırıcı egzersizler hemen başlanır. Breyse gerek yoktur. Altıncı haftada tam yük vermeye izin verilir.

\section{Literatür Değerlendirmesi}

İnstabil dizde tek başına PTO ile ilgili yeterli literatür bilgisi yoktur. Dejeneratif değişikliğe bağlı ağrısı ön planda olan küçük hasta gruplarında genel olarak iyi sonuçlar bildirilmiştir. Fowler ve ark., lateral kapalı kama osteotomisi (LKKO) uyguladıkları yedi hastanın kısa izlemlerinde ağııda azalma, stabilite ve fonksiyonda iyileşme bildirmişlerdir. ${ }^{[27]}$ Lattermann ve Jacob, LKKO ile 11 hastanın 10'unda ağrıda önemli iyileşme ve instabilitede ilerleme olmadığını bildirmişlerdir. ${ }^{[11]}$ Bir başka çalışmada erken medial artrit, ÖÇB yetersizliği ve varus dizilim bozukluğu olan ortalama 43 yaşındaki 29 hastaya ÖÇB yaralanmasından ortalama 14 yıl sonra ÖÇBR ile kombine YTO yapılmış. Hastaların altı yıllık izlemlerinde \%80'inin spora yeniden döndüğü, $\% 50$ 'sinin yarışmacı sporlarla ilgilendiği, \%97'sinde instabilite şikâyetinin kalmadığı, 
\%72'sinin ağrısının iyileştiği ve IKDC (International Knee Documentation Commitee) evrelemesine göre osteoartiritte radyolojik ilerleme saptanmadığı bildirilmiştir. ${ }^{[28]} \mathrm{Eş}$ zamanlı ÖÇBR ve PTO yapılan çalışmaları içeren sistematik derlemede anterior stabilitenin sağlandığı, semptomlarda iyileşmenin olduğu ve rekreasyonel sporlara dönüşün iyi olduğu bildirilmiştir. ${ }^{[29]}$

Kombine cerrahide karşılaşılan sorunlar izole ÖÇBR veya izole PTO'nun tek başına yapıldıklarında karşılaşılanlardan farklı değildir. Komplikasyon oranı çok düşük değildir (\%0-30). Derin ven trombozu, yüzeyel yara enfeksiyonu ve hematom en sık görülen komplikasyonlardır. Osteotomi yerinde kaynamada gecikme ve hareket kısıtlılığı da bildirilmiştir.[13]
Bezmialem Üniversitesi Tıp Fakültesi Ortopedi ve Travmatoloji Kliniği olarak 20 hastaya eş zamanlı ÖÇB rekonstrüksiyonu ve PTO uyguladık. ÖÇB rekonstrüksiyonu için bir olgu dışında tüm olgularda hamstring otogrefti kullandık. Dört hastaya revizyon ÖÇBR ile birlikte PTO uyguladık. Revizyon uyguladığımız olguların birinde kemik-patellar tendon-kemik grefti uyguladık. Osteotomilerin hepsi medial açık kama osteotomisidir. Hiçbir hastamızda kemik grefti uygulamadık. Bir ile dört yıllık izlemde hiçbir olguda başka cerrahiye gereksinim olmamış ve hastaların subjektif IKDC (International Knee Documentation Commitee) skorlarında belirgin iyileşme sağlanmıştır (Şekil 4).
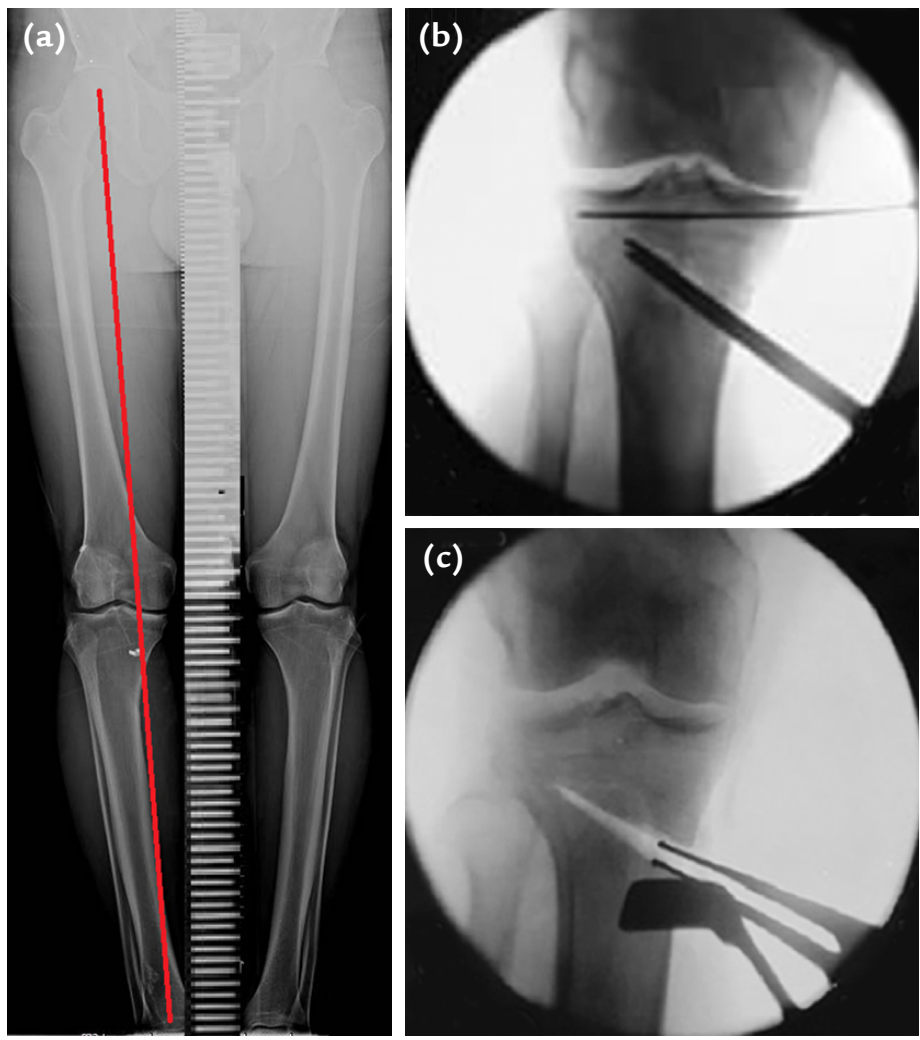

Şekil 4. a-g. ÖÇB rekonstrüksiyonu yetersizliği ve varus osteoartriti olan hastada karşı dizden alınan hamstring tendon grefti ile revizyon ÖÇB rekonstrüksiyonu ve proksimal tibial osteotomi: Ameliyat öncesi grafi (a). Skopi kontrolünde osteotominin yapılması (b, c). Plağın proksimal anteriordaki deliği ile tibial tünelin ilişkisi (d). Greftin geçirilmesi ve tespiti (e, f). Ameliyat sonrası grafi (g).
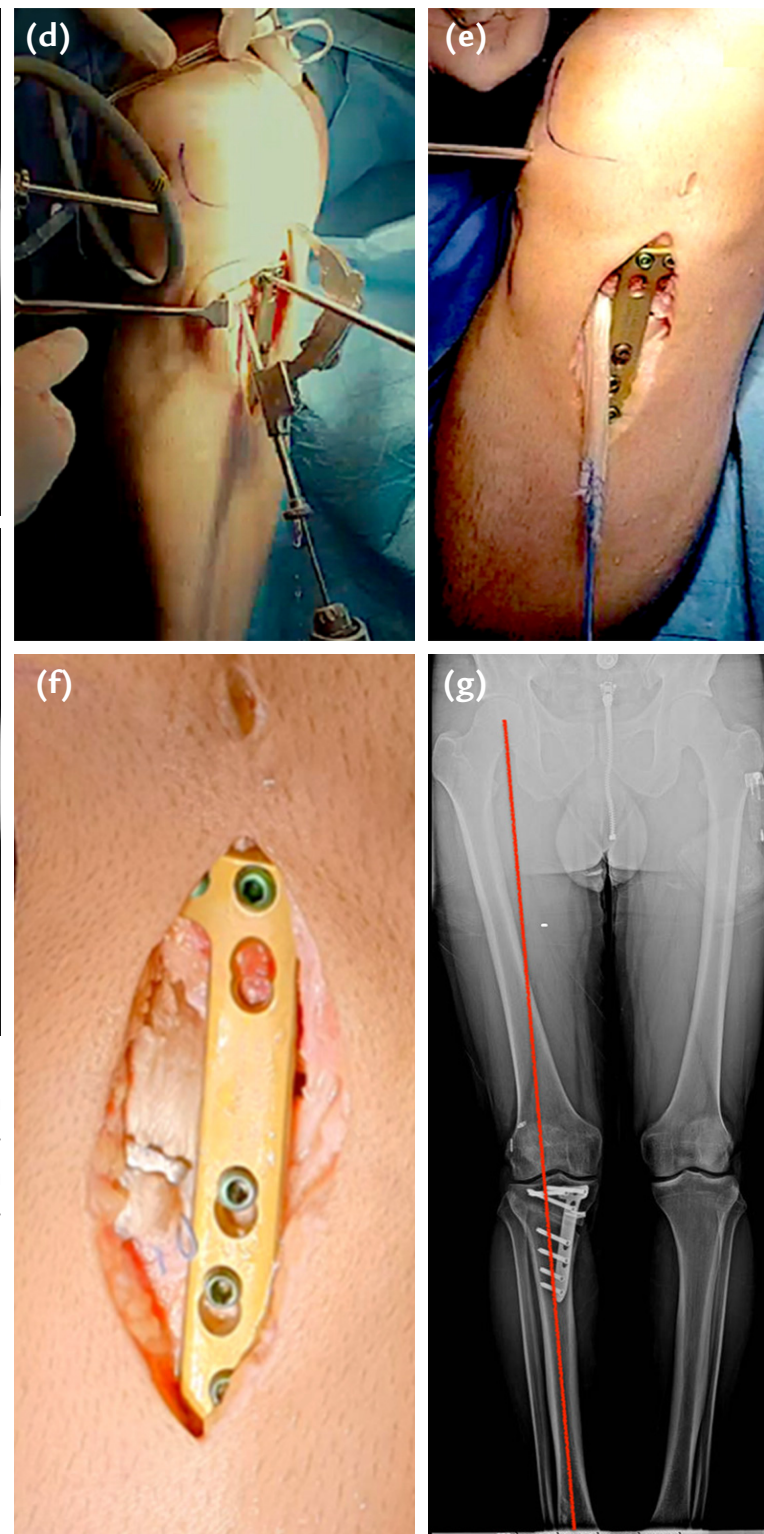


\section{SONUÇ}

Anstabil dizilim bozukluğu ve artrozu olan dizlerin tedavisi güçtür. ÖÇB veya $A C ̧ B$ yetersizliğine göre tibial eğimi değiştirmeye dikkat ederek bazı hastalarda tek başına PTO stabilize edici etkiye sahip olabilir. Eş zamanlı PTO ile kombine ÖÇBR yapılmasında başarılı sonuçlar bildirilmiştir. Ancak mevcut çalışmalarda hasta grubunun homojen olmayışı ve az sayıda olması nedeniyle sonuç çıkarmak zordur. Modern cerrahi teknikler, stabil tespit ve erken rehabilitasyon yöntemleri ile eş zamanlı girişimlerin komplikasyon oranları çok düşüktür. Kombine girişim artrozun ilerlemesini yavaşlatır, ağrı ve instabiliteyi gidermekte etkilidir.

\section{KAYNAKLAR}

1. Gillquist J, Messener K. Anterior ligament reconstruction and the long term incidence of gonarthrosis. Sports Med 1999;27(3):143-56. Crossref

2. Friel NA, Chu CR. The role of ACL injury in the development of posttraumatic knee osteoarthritis. Clin Sports Med 2013;32(1):1-12. Crossref

3. Louboutin H, Debarge R, Richou J, Selmi TAS, Donell ST, Neyret P, Dubrana F. Osteoarthritis in patients with anterior cruciate ligament rupture: a review of risk factors. Knee 2009;16(4):239-44. Crossref

4. Thompson WO, Fu FH. The meniscus in the cruciate deficient knee. Clin Sports Med 1993;12(4):771-96.

5. Cohen M, Amaro J, Ejnisman B, Carvalho RT, Nakano KK, Peccin MS, Teixeira R, Laurino CFS, Abdalla RJ. Anterior cruciate ligament reconstruction after 10 to 15 years: association between meniscectomy and osteoarthritis. J Arthrosc Rel Surg 2007;23(6):629-34.

6. Van de Pol GJ, Arnold MP, Verdonschot N, van Kampen A. Varus alignment leads to increased forces in the anterior cruciate ligament. Am J Sports Med 2009;37(3):481-7. Crossref

7. Yoon $\mathrm{KH}$, Lee $\mathrm{SH}$, Bae DK, Park SY, Oh H. Does varus alignment increase after medial meniscectomy? Knee Surg Sports Traumatol Arthrosc 2013;21(9):2131-6. Crossref

8. Elser F, Imhoff AB. Combined High Tibial Osteotomy, Anterior Cruciate Ligament Reconstruction, and Cartilage Transplantation in Young Athletes. Oper Tech Orthop 2007;17(1):46-50. Crossref

9. Imhoff $A B$, Linke RD, Agneskirchner J. Corrective osteotomy in primary varus, double varus and triple varus knee instability with cruciate ligament replacement. Orthopade 2004;33(2):201-7. Crossref

10. Lohmander LS, Englund PM, Dahl LL, Roos EM. The longterm consequence of anterior cruciate ligament and meniscus injuries: Osteoarthritis. Am J Sports Med 2007;35(10):175669. Crossref

11. Lattermann C, Jakob RP. High tibial osteotomy alone or combined with ligament reconstruction in anterior cruciate ligament-deficient knees. Knee Surg Sports Traumatol Arthrosc 1996;4(1):32-8. Crossref

12. Benjamin Herman V, Robert Griffin J. High tibial osteotomy in the ACL deficient knee with medial compartment osteoarthritis. J Orthop Traumatol 2016;17(3):277-85. Crossref
13. Noyes FR, Barber-Westin SD, Hewett TE. High tibial osteotomy and ligament reconstruction for varus angulated anterior cruciate ligament- deficient knees. Am J Sports Med 2000;28(3):282-96. Crossref

14. Stride D, Wang J, Horner NS, Alolabi B, Khanna V, Khan $M$. Indications and outcomes of simultaneous high tibial osteotomy and $\mathrm{ACL}$ reconstruction. Knee Surg Sports Traumatol Arthrosc 2019;27(4):1320-31. Crossref

15. Dejour H, Neyret P, Boileau P, Donell ST. Anterior cruciate reconstruction combined with valgus tibial osteotomy. Clin Orthop Relat Res 1994;(299):220-8. Crossref

16. Gupta A, Tejpal T, Shanmugaraj A, Horner NS, Simunovic N, Duong A, Ayeni OR. Surgical Techniques, Outcomes, Indications, and Complications of Simultaneous High Tibial Osteotomy and Anterior Cruciate Ligament Revision Surgery: A Systematic Review. HSS Jrnl 2019;15(2):176-84. Crossref

17. Bonasia D, Governale G, Spolaore S, Rossi R, Amendola A. High tibial osteotomy. Curr Rev Musculoskelet Med 2014;7(4):292-301. Crossref

18. Kim SJ, Moon HK, Chun YM, Chang WH, Kim SG. Is correctional osteotomy crucial in primary varus knees undergoing anterior cruciate ligament reconstruction. Clin Orthop Relat Res 2011;469(5):1421-6. Crossref

19. Robin JG, Neyret P. High tibial osteotomy in knee laxities: Concepts review and results. EFORT Open Rev 2016;1(1):311. Crossref

20. Naudie DDR, Amendola A, Fowler PJ. Opening wedge high tibial osteotomy for symptomatic hyperextension-varus thrust. Am J Sports Med 2004;32(1):60-70. Crossref

21. Phisitkul P, Wolf BR, Amendola A. Role of high tibial and distal femoral osteotomies in the treatment of lateral-posterolateral and medial instabilities of the knee. Sports Med Arthrosc 2006;14(2):96-104. Crossref

22. Cantin O, Magnussen R, Corbi F, Servien E, Neyret P, Lustig $\mathrm{S}$. The role of high tibial osteotomy in the treatment of knee laxity: a comprehensive review. Knee Surg Sports Traumatol Arthrosc 2015;23(10):3026-37. Crossref

23. Arthur A, LaPrade RF, Agel J. Proximal tibial opening wedge osteotomy as the initial treatment for chronic posterolateral corner deficiency in the varus knee: a prospective clinical study. Am J Sports Med 2007;35(11):1844-50. Crossref

24. Petrigliano FA, Suero EM, Voos JE, Pearle AD, Allen AA. The effect of proximal tibial slope on dynamic stability testing of the posterior cruciate ligament and posterolateral corner deficient knee. Am J Sports Med 2012;40(6):1322-8. Crossref

25. Agneskirchner JD, Hurschler C, Stukenborg-Colsman C, Imhoff $A B$, Lobenhoffer P. Effect of high tibial flexion osteotomy on cartilage pressure and joint kinematics: a biomechanical study in human cadaveric knees. Arch Orthop Trauma Surg 2004;124(9):575-84. Crossref

26. Todd MS, Lalliss S, Garcia E, DeBerardino TM, Cameron KL. The relationship between posterior tibial slope and anterior cruciate ligament injuries. Am J Sports Med 2010;38(1):63-7. Crossref

27. Fowler PJ, Kirkley A, Roe J. Osteotomy of the proximal tibia in the treatment of chronic anterior cruciate ligament insufficiency. J Bone Jt Surg Suppl 1994;76B.

28. Trojani C, Elhor $\mathrm{H}$, Carles M, Boileau P. Anterior cruciate ligament reconstruction combined with valgus high tibial osteotomy allows return to sports. Orthop Traumatol Surg Res 2014;100(2):213-16. Crossref

29. Li Y, Zhang H, Zhang J, Li X, Song G, Feng H. Clinical outcome of simultaneous high tibial osteotomy and anterior cruciate ligament reconstruction for medial compartment osteoarthritis in young patients with anterior cruciate ligament-deficient knees: a systematic review. Arthroscopy 2015;31(3):507-19. Crossref 